Original Paper http://ajol.info/index.php/ijbcs http://indexmedicus.afro.who.int

\title{
Analyse de l'importance socio-économique de l'aviculture familiale dans le Département de Salemata au Sénégal
}

\author{
Grégoire NAHIMANA ${ }^{1 *}$, Walter OSSEBI ${ }^{2}$, Ayao MISSOHOU ${ }^{3}$ et \\ Simplice Bosco AYSSIWEDE ${ }^{3}$
}

\author{
${ }^{I}$ Faculté d'Agronomie et de Bio Ingénierie, Université du Burundi, BP 1550 Bujumbura, Burundi. \\ ${ }^{2}$ Service d'Economie Rurale et Gestion, Ecole Inter-Etats des Sciences et Médecine Vétérinaires de Dakar \\ (EISMV), BP 5077 Dakar, Sénégal. \\ ${ }^{3}$ Service de Zootechnie-Alimentation, Ecole Inter-Etats des Sciences et Médecine Vétérinaires de Dakar \\ (EISMV), BP 5077 Dakar, Sénégal. \\ *Auteur correspondant ; E-mail: gregoirenahi@yahoo.fr
}

\section{RÉSUMÉ}

Dans le but d'analyser la contribution du poulet indigène à la génération des revenus et à la sécurité alimentaire dans un ménage, une enquête longitudinale d'une année a été réalisée dans 45 exploitations avicoles du Département de Salémata (Sénégal). Les résultats ont montré que les effectifs de poules sont passés de 17,1 à 23,4 poules. Les causes les plus importantes de variation des effectifs des poules dans un ménage ont été la consommation $(25,5 \%)$, les ventes $(31,22 \%)$ et les pertes $(38,68 \%)$. Les pertes ont été causées en grande partie par les rapaces $(21,61 \%)$ et les chats $(65,80 \%)$. Quant aux circonstances de consommation des poules, les plus importantes ont été les festivités religieuses $(37,97 \%)$ et de fin d'année $(26,06 \%)$ ainsi que les consommations domestiques $(14,34 \%)$ ou d'accueil d'un hôte $(11,52 \%)$. Les œufs pondus $(10,2)$ sont presque tous couvés $(10,1)$. Les taux d'éclosion et de mortalité des poussins ont été de $81,36 \%$ et de $47,11 \%$. Le rapport profit sur investissement, consommations internes du ménage incluses, a été de 4,05. Les consommations moyennes annuelles d'œufs et de poules par ménage ont été de 56,7 œufs et de 48 poules dans un ménage de taille moyenne de 7 personnes. Il ressort de cette étude que l'aviculture familiale constitue un moyen d'amélioration de la sécurité alimentaire et d'atténuation de la pauvreté. Des recherches doivent être faites pour améliorer la protection des poussins, l'alimentation et la santé des oiseaux.

(C) 2019 International Formulae Group. All rights reserved

Mots clés : Aviculture familiale, sécurité alimentaire, lutte contre la pauvreté, socio-économie, Sénégal.

\section{Socio-economic importance of family poultry in Senegal}

\begin{abstract}
A one-year longitudinal survey was carried out in 45 poultry farms in Salémata Department (Senegal) to show the contribution of family poultry to poverty alleviation and food security in a household. The results showed that chicken numbers increased from 17.1 to 23.4 hens. The most important causes of variation in hen numbers in a household were consumption $(25.5 \%)$, losses $(38.68 \%)$ and sales $(31.22 \%$ hens). The most frequent causes of mortality were predators (Raptors: $21.61 \%$ and cats: $65.80 \%$ ). The most important
\end{abstract}


circumstances of consumptions were religious holidays $(37.97 \%)$ and year-end $(26.06 \%)$, the household $(14.34 \%)$ or to welcome a host $(11.52 \%)$. The eggs laid (10.2) are almost all incubated (10.1). The hatching rate and chicks' mortality were $81.36 \%$ and $47.11 \%$, respectively. The return (profit/investment) with family consumption was 4.05. The average annual consumption of eggs and chickens per household was 56.7 eggs and 48 chickens in a household size of 7 people. This study shows that family poultry constitute an improving food security and poverty alleviation. Researches are to be done to improve chick protection, feeding and bird health.

(C) 2019 International Formulae Group. All rights reserved

Keywords: Family poultry, Senegal, socio-economy, food security, poverty alleviation.

\section{INTRODUCTION}

La population sénégalaise a évolué de 9855 millions d'habitants en 2002 (59,3\% de ruraux contre $40,7 \%$ de citadins) à 13508715 habitants en 2013 (55\% de ruraux contre $45 \%$ de citadins), soit un taux d'urbanisation de 4,3\% (MEFP, 2014). Cette croissance démographique s'est accompagnée de l'augmentation des besoins alimentaires alors que la population souffre déjà d'une malnutrition protéique en qualité et en quantité. Ainsi, le pays doit entreprendre des mesures pour subvenir aux besoins de cette population, d'où la nécessité d'accroître la disponibilité en protéines d'origine animale s'impose (Niang et Mbaye, 2015).

Etant donné la vulnérabilité du gros bétail aux aléas climatiques et sanitaires, le développement de l'élevage devrait se faire essentiellement par les animaux à cycle court et en premier lieu la volaille, source importante de protéines (Hofman, 2000) et un élément fondamental dans la stratégie de lutte contre la pauvreté et la malnutrition (Guèye, 2004).

En effet, les effectifs de la volaille traditionnelle sont passés de 23,2 millions de têtes en 2006 à 26,93 millions en 2012 et l'aviculture familiale fournit $14,5 \%$ de la consommation totale de viande au Sénégal (DIREL, 2013).

Ainsi, du fait de ses nombreuses potentialités (espèce à cycle court, de production plus facile et nécessitant peu d'investissements, accessible à tous), l'aviculture familiale occupe aujourd'hui une place importante dans les stratégies de développement et de lutte contre la pauvreté dans la plupart des pays en voie de développement (Sonaiya et Swan, 2004).

D'un autre côté, chaque ménage en milieu rural élève un petit cheptel de poules indigènes en divagation. Cette volaille de basse-cour enrichit les familles laborieuses rurales et sans-terre, les plus pauvres à travers une approche holistique et indépendante non seulement en termes d'amélioration des revenus, d'emplois et du statut nutritionnel mais aussi en termes de stimulation du développement communautaire, de renforcement de l'aspect genre et de protection de l'environnement (Sarkar et Bell, 2006).

Les études réalisées sur la socioéconomie de l'aviculture familiale ont été sous forme d'enquête de courte durée d'où l'intérêt de cette étude.

L'objectif de ce travail est d'analyser le rôle joué par l'aviculture familiale dans l'amélioration de la sécurité alimentaire et la lutte contre la pauvreté des populations rurales et démunies.

\section{MATERIEL ET METHODES \\ Période et milieu d'étude}

L'étude a été menée de septembre 2014 à août 2015 dans le département de Salémata, situé au Sénégal oriental, caractérisé par un climat subguinéen, avec une saison de pluies unique et courte de mai à septembre, et une saison sèche longue d'octobre à avril. L'humidité relative est très 
élevée entre août et octobre (97\%) et baisse pour atteindre une valeur minimale voisine de $10 \%$ entre janvier et mars. La température moyenne est de $28,3{ }^{\circ} \mathrm{C}$ avec des précipitations annuelles moyennes de 1300 $\mathrm{mm}$.

\section{Exploitations avicoles et leur suivi}

L'étude longitudinale a porté sur 45 exploitations réparties dans cinq communes de Salémata retenues suivant les critères développés dans Nahimana et al. (2017).

Un questionnaire préétabli, testé et validé a été administré aux éleveurs au début de cette enquête. Les informations à renseigner concernaient les caractéristiques socioéconomiques de l'éleveur (sexe, âge, niveau de scolarisation, situation matrimoniale, objectifs visés, sources de revenus, structure familiale), les caractéristiques et la description de l'exploitation (composition du cheptel avicole, conduite de l'élevage avicole, performances de production et de la productivité, exploitation du cheptel).

Les aviculteurs sélectionnés pour le suivi avaient bénéficié d'une poussinière et de ses deux mangeoires dont les caractéristiques sont décrites par Nahimana et al. (2015).

Au début de l'essai, le cheptel avicole a été vacciné contre les maladies de Gumboro et de Newcastle après avoir été compté par classe d'âge et par sexe chez les adultes. L'opération de comptage a été également faite au début et à la fin du suivi. La vaccination contre la maladie de Newcastle a été renouvelée tous les quatre mois.

\section{Collecte des données}

La collecte des données de suivi a été réalisée une fois toutes les deux semaines dans chaque exploitation. Les paramètres liés aux mouvements des volailles dont les entrées et les sorties (mortalités, vente/achats, confiage, consommations, dons, ...) dans une exploitation ont été enregistrés. Pour les ventes, l'utilisation des revenus issus de cette activité a été mentionnée.

Les dates de début de ponte et d'éclosion des poules de ces exploitations ont été enregistrées. Le nombre d'œufs pondus, consommés et couvés, et de poussins à l'éclosion ont été enregistrés. La pesée des poussins a été effectuée à l'éclosion, et à un, trois et six mois d'âge.

\section{Analyse des données}

Les données collectées ont été enregistrées avec le tableur Excel (Microsoft Office 2007) et synthétisées en termes de fréquences, pourcentages et moyennes avec le logiciel SPSS version 16 (SPSS, Chicago, IL, USA). L'analyse de ces données a permis de calculer les paramètres zootechniques suivants:

- taux d'éclosion $(\%)=100 \times($ nombre d'œufs éclos / nombre d'œufs couvés) ;

- nombre moyen d'œufs par poule par an = somme totale des œufs pondus par toutes les poules par an / nombre total de poules ;

- taux de survie des poussins $(\%)=100 \times$ (nombre total de poussins vivants / nombre de poussins éclos).

La méthode de Goodger (2002) a été utilisée pour évaluer économiquement l'importance de l'aviculture familiale :

- Revenus $=$ prix de vente des poules + prix de vente des œufs ;

- Charges $=$ somme des charges (alimentaires, vaccination, médication, poussinière et son petit matériel);

- Profit net $=$ Revenus - charges ;

- Rendement $=$ Profit net $/$ charges

\section{RESULTATS}

\section{Statut socioéconomique des aviculteurs}

Les aviculteurs suivis sont en majorité mariés $(95,6 \%)$ et de sexe féminin $(57,8 \%)$. Ils sont majoritairement musulmans $(86,7 \%)$ situés dans la classe d'âge compris entre 31 et 49 ans $(53,33 \%)$. Leur niveau d'instruction est assez bas $(60 \%$ d'analphabètes et d'alphabétisés). Les objectifs principaux de la 
production avicole sont la vente d'oiseaux $(51,11 \%)$ et l'autoconsommation (40\%). Même si différentes activités sont pratiquées par ces aviculteurs, la quasi-totalité d'entre eux $(57,11 \%)$ sont des agriculteurs (Tableau $1)$.

\section{Cheptel}

L'élevage de poules dans les cinq communes du département de Salémata est presque mono spécifique du fait qu'il est peu associé à celui d'autres oiseaux (canards, pintades, pigeons, dindons). La taille moyenne du cheptel de poules est de 17,1 $\pm 10,6$ au début et $23,4 \pm 14,8$ poules à la fin du suivi par ménage. Il se compose de $1,8 \pm 1,3$ et de $1,0 \pm 0,0$ mâles adultes et de $4,8 \pm 3,4$ et de $6,4 \pm 3,3$ femelles, respectivement, au début et à la fin du suivi. Le nombre de poussins a varié de 6,3 au début à 8,7 à la fin du suivi. Les variations ont été positives sauf pour les mâles adultes $(-44,4 \%)$, le sex ratio $(-46 \%)$ ainsi que le pourcentage de la race locale (71,3\%) (Tableau 2).

Les prélèvements à l'origine de la variation des effectifs des poules par ménage sont constitués par les festivités marquant la naissance de bébé, l'autoconsommation, des pertes accidentelles de poules, les ventes, l'achat de nouveaux sujets, les dons et les sacrifices/rituels. Les causes les plus importantes de déstockage des poules sont les festivités marquant les naissances $(98,89 \%)$, les pertes $(38,68 \%)$, les ventes $(31,22 \%)$ et l'autoconsommation $(26,19 \%)$ (Tableau 3).

Les causes de mortalité des poules dans un ménage sont les prédateurs (rapaces, chats), les maladies infectieuses et parasitaires. Les plus importantes sont principalement les rapaces $(21,61 \%)$ et les chats $(65,8 \%)$. Les autres causes (maladies infectieuses et parasitaires, inconnues) sont faiblement représentées (12,59\%) (Tableau 4).

Les différentes circonstances de l'autoconsommation des poules dans un ménage sont rapportées au Tableau 5.
Il ressort de ce tableau que les circonstances de l'autoconsommation des poules du pays dans un ménage sont les festivités religieuses $(36,97 \%)$ et de fin d'année $(26,06 \%)$, la consommation familiale (14,31\%) ou l'accueil d'un hôte $(11,52 \%)$ ou tout simplement les pratiques rituelles $(11,11 \%)$.

\section{Performances et profitabilité des poules en aviculture familiale dans un ménage à Salémata (Sénégal)}

Le Tableau 6 montre les performances, les charges (alimentaire, vaccination, médication) et le revenu généré par la vente des poules locales ainsi que le rapport investissement sur profit dans un ménage.

Il ressort de ce tableau que le nombre d'œufs varie de 4 à 10,2 et de 4 à 10,1 , respectivement, pour les œufs pondus et couvés. Le taux moyen d'éclosion est de $81,36 \%$ et varie de 22,22 à $100 \%$. Le taux moyen de mortalité $(39,11 \%)$ varie de 19,57 à $84,26 \%$. Les charges sont liées aux coûts des aliments, de la vaccination, de la médication, de la poussinière, des mangeoires et de l'abreuvoir porte-bouteilles alors que les entrées sont constituées des ventes des œufs et des poules. Le revenu par ménage est 157032 Fcfa tandis que le profit net annuel par ménage est de 99995 Fcfa. Tenant compte du coût d'investissement de 57037 Fcfa, le rapport investissement sur profit est de 1 : 1,75 si les consommations sont exclues et de $1: 4,05$ dans le cas contraire.

\section{Sécurité nutritionnelle des ménages ruraux et le rôle des poules indigènes}

Le Tableau 7 montre les

consommations moyennes annuelles d'œufs et de poules par ménage.

Les consommations moyennes annuelles d'œufs et de poules par ménage sont de 56,7 œufs et de 48 poulets, soit 8,1 œufs et 7,1 poules individuellement dans un ménage de taille égale à 7 personnes. 
Tableau 1: Statut socio-économique des aviculteurs suivis à Salémata (Sénégal).

\begin{tabular}{lcc}
\hline Paramètres et caractéristiques & Effectif & Pourcentage (\%) \\
\hline Sexe et situation matrimoniale (\%) : & 26 & 57,8 \\
Féminin & 19 & 42,2 \\
Masculin & 43 & 95,6 \\
Marié & 2 & 4,4 \\
Célibataire & & \\
Age (années) : & 15 & 33,33 \\
$<31$ & 24 & 53,33 \\
$31-50$ & 6 & 13,33 \\
$>50$ & & \\
Religion & 39 & 86,7 \\
Musulman & 6 & 13,3 \\
Chrétien & & \\
Niveau d'instruction : & 27 & 60 \\
Analphabètes et alphabétisés & 2 & 4,4 \\
Primaire & 13 & 28,9 \\
Secondaire & 3 & 6,7 \\
Universitaire & & \\
Objectif principal de la production (\%) : & 18 & 40 \\
Autoconsommation & 23 & 51,11 \\
Vente & 4 & 8,81 \\
Socio-culture (sacrifice, échange, accueil hôte) & & \\
Activités principales (\%) : & 26 & 57,8 \\
Agriculture & 5 & 11,11 \\
Elevage au sens large & 14 & 31,1 \\
Autres professions (Agro-éleveur, profession libérale, & \\
fonctionnaire) & & \\
\hline
\end{tabular}

Tableau 2 : Espèces et catégories d'oiseaux élevés à Salémata (Sénégal).

\begin{tabular}{|c|c|c|c|}
\hline $\begin{array}{lll}\begin{array}{l}\text { Espèce } \\
\text { (moyenne) }\end{array} & \text { par } & \text { ménage } \\
\end{array}$ & $\begin{array}{l}\text { Début du } \\
\text { suivi }\end{array}$ & Fin du suivi & Variations (\%) \\
\hline Canards & 0,5 & 0,1 & -80 \\
\hline Pintades & 0,2 & 0,2 & 0 \\
\hline Pigeons & 0,0 & 0,2 & +200 \\
\hline Dindons & 0,0 & 0,0 & 0,0 \\
\hline Poules & $17,1 \pm 10,6$ & $23,4 \pm 14,8$ & $+36,8$ \\
\hline Mâles adultes & $1,8 \pm 1,3$ & $1,0 \pm 0,0$ & $-44,4$ \\
\hline Femelles adultes & $4,8 \pm 3,4$ & $6,4 \pm 3,2$ & $+33,3$ \\
\hline Mâles en croissance & $1,6 \pm 2,5$ & $3,8 \pm 3,4$ & $+137,5$ \\
\hline Femelles en croissance & $2,6 \pm 3,6$ & $3,5 \pm 2,8$ & $+34,6$ \\
\hline Poussins & $6,3 \pm 5,6$ & $8,7 \pm 9,9$ & $+38,1$ \\
\hline Mâle/femelle & $1 / 2,7$ & $1 / 5$ & -46 \\
\hline Race locale $(\%)$ & 91,8 & 20,5 & $-71,3$ \\
\hline Race exotique* $(\%)$ & 1,7 & 4,7 & $+3,0$ \\
\hline Croisés $(\%)$ & 6,6 & 74,4 & $+67,8$ \\
\hline
\end{tabular}


Tableau 3 : Causes de la variation des effectifs des poules dans un ménage à Salémata (Sénégal).

\begin{tabular}{lcccccc}
\hline Rubriques & \multicolumn{5}{c}{ Causes de la variation de l'effectif des poules } \\
\cline { 2 - 7 } & Naissances & Consommation & Pertes & Ventes & Achats & Don \\
\hline $\begin{array}{l}\text { Effectif de poules } \\
\text { déstockées/événement }\end{array}$ & 186,9 & 49,5 & 73,1 & 59,2 & 2,1 & 0,9 \\
$\begin{array}{l}\text { Pourcentage par rapport } \\
\text { aux ajouts }\end{array}$ & 98,89 & 26,19 & 38,68 & 31,22 & 1,11 & 0,69 \\
\hline
\end{tabular}

Tableau 4 : Causes des mortalités des poules par ménage à Salémata (Sénégal).

\begin{tabular}{lccccccc}
\hline Rubrique & \multicolumn{6}{c}{ Cause des mortalités des poulets } & \multicolumn{2}{c}{ Total } \\
\cline { 2 - 7 } & Rapaces & Chats & Newcastle & Variole & Parasitoses & Inconnues & \\
\hline Effectif & 15,8 & 48,1 & 3,9 & 1,3 & 0,8 & 3,2 & 73,1 \\
Pourcentage & 21,61 & 65,80 & 5,34 & 1,78 & 1,09 & 4,38 & 100 \\
\hline
\end{tabular}

Tableau 5 : Circonstances de l'autoconsommation des poules locales dans un ménage à Salémata (Sénégal).

\begin{tabular}{lcccccc}
\hline \multirow{2}{*}{ Rubriques } & \multicolumn{4}{c}{ Circonstances de l'autoconsommation des poulets } & Total \\
\cline { 2 - 6 } & $\begin{array}{c}\text { Festivités de } \\
\text { fin d'année }\end{array}$ & $\begin{array}{c}\text { Festivités } \\
\text { religieuses }\end{array}$ & $\begin{array}{c}\text { Accueil } \\
\text { d'un hôte }\end{array}$ & $\begin{array}{c}\text { Pratique } \\
\text { rituelle }\end{array}$ & $\begin{array}{c}\text { Consommatio } \\
\text { n familiale }\end{array}$ & \\
\hline Effectif des poules & 12,9 & 18,3 & 5,7 & 5,5 & 7,1 & 49,5 \\
Pourcentage & 26,06 & 36,97 & 11,52 & 11,11 & 14,34 & 100 \\
\hline
\end{tabular}

Tableau 6 : Performances et profitabilité des poules en aviculture familiale dans un ménage à Salémata (Sénégal).

\begin{tabular}{lccc}
\hline Paramètres & Minimum & Maximum & Moyenne \\
\hline Eufs pondus par poule & 4 & 18 & 10,2 \\
Eufs couvés & 4 & 16 & 10,1 \\
Eufs éclos & 2 & 15 & 8,4 \\
Taux d'éclosion (\%) & 22,22 & 100 & 81,36 \\
Taux de mortalité (\%) à 6 mois & 19,57 & 84,26 & 39,11 \\
\hline Charges alimentaires par an & Quantités & PU (FCFA) & Coût total (FCFA) \\
\hline Son de maïs (kg) & 65,44 & 25 & 1636 \\
Grains de maïs (kg) & 41,58 & 200 & 8316 \\
Mil (kg) & 7,23 & 150 & 1085 \\
Aliment industriel (kg) & 0,5 & 500 & 250 \\
\hline Sous total 1 & & & $\mathbf{1 1 2 8 7}$ \\
\hline
\end{tabular}




\begin{tabular}{|c|c|c|c|}
\hline \multicolumn{4}{|l|}{$\begin{array}{l}\text { Charges de vaccination et de } \\
\text { médication par an }\end{array}$} \\
\hline Vaccin Newcastle (dose) & 200 & 25 & 5000 \\
\hline Vaccin Variole (dose) & 50 & 15 & 750 \\
\hline Déparasitant (sachet) & 2 & 1500 & 3000 \\
\hline Antibiothérapie (sachet) & 2 & 2000 & 4000 \\
\hline Vitaminothérapie (sachet) & 1 & 3000 & 3000 \\
\hline Sous total 2 & & & 15750 \\
\hline \multicolumn{4}{|l|}{ Charges fixes } \\
\hline Poulailler & 1 & 2500 & 2500 \\
\hline Poussinières & 1 & 13000 & 13000 \\
\hline Mangeoire pour poule & 1 & 2500 & 2500 \\
\hline Mangeoire pour poussins & 1 & 2750 & 2750 \\
\hline Abreuvoir porte-bouteilles & 1 & 1750 & 1750 \\
\hline Sous total 3 & & & 22500 \\
\hline Amortissement & & & 7500 \\
\hline Total des charges annuelles & & & 57037 \\
\hline \multicolumn{4}{|l|}{ Ventes } \\
\hline CEufs & 8 & 100 & 800 \\
\hline Poulets & 59 & 2648 & 156232 \\
\hline \multirow[t]{2}{*}{ Revenus par ménage } & \multicolumn{2}{|c|}{ Consommations du ménage exclues } & 157573 \\
\hline & \multicolumn{2}{|c|}{ Consommations du ménage incluses } & 288108 \\
\hline \multirow{2}{*}{ Profit net par ménage } & \multicolumn{2}{|c|}{ Consommations du ménage exclues } & 99995 \\
\hline & \multicolumn{2}{|c|}{ Consommations du ménage incluses } & 231071 \\
\hline \multirow[t]{2}{*}{ Rapport : investissement/profit net } & \multicolumn{2}{|c|}{$\begin{array}{l}\text { Consommation internes du ménage } \\
\text { exclues }\end{array}$} & $1: 1,75$ \\
\hline & \multicolumn{2}{|c|}{$\begin{array}{l}\text { Consommations internes du ménage } \\
\text { incluses }\end{array}$} & $1: 4,05$ \\
\hline
\end{tabular}

Tableau 7 : Consommations moyennes annuelles d'œufs et de viande de poules chez les bénéficiaires à Salémata (Sénégal).

\begin{tabular}{lccc}
\hline Paramètres & Minimum & Maximum & Moyenne \\
\hline Effectif de personnes par ménage & 4 & 16 & $7 \pm 2,5$ \\
Consommation d'œufs/ménage/an & 45,8 & 89,4 & $56,7 \pm 12,9$ \\
Consommation d'œufs/individu/an & 6,3 & 13,9 & $8,1 \pm 3,5$ \\
Consommation de poule/ménage/an & 36,4 & 75,7 & $49,5 \pm 11,3$ \\
Consommation de poule/individu/an & 4,7 & 11,5 & $7,1 \pm 2,4$ \\
\hline
\end{tabular}




\section{DISCUSSION}

\section{Statut socioéconomique des aviculteurs}

Il ressort de cette étude que les propriétaires des exploitations avicoles suivies étaient majoritairement des femmes. Cela confirme les constats de nombreux auteurs en Afrique de l'Ouest et de l'Est (Tadelle et Ogle, 2001 ; Muchadeyi et al., 2004 ; Moula et al., 2012 ; Fall et al., 2016) et en Asie (Riise et al., 2005) selon lesquels, les femmes prédominent en aviculture familiale et que les hommes sont propriétaires des ruminants. La prédominance féminine pourrait être liée au fait que les femmes s'occupent principalement des activités ménagères et veillent le plus aux soins et à la conduite des volailles qui nécessitent peu d'investissements par rapport au plus grand bétail (Copland et Alders, 2005). Par ailleurs, et dans la plus part des cas, elles sont en général loin des sources d'information, contrairement aux hommes qui sont présents dans tous les fora et séminaires (Guèye, 2000, 2003).

L'objectif principal de production (autoconsommation et vente) des aviculteurs suivis dans la présente étude corrobore les observations faites par Moula et al. (2012) et Dinka et al. (2010).

Le nombre d'œufs pondus par couvée dans la présente étude se situe dans l'intervalle de 8 à 20 œufs rapporté par Benabdeljelil et Arfaoui (2001). Tous les œufs pondus sont couvés en quasi-totalité en accord avec Sonaiya et Swan (2004) et van Eekeren et al. (2004). D'après ces auteurs, presque tous les œufs sont quasiment destinés à la reproduction dans le but de renouveler le cheptel. Par ailleurs, le nombre d'œufs annuellement consommés ( 8 œufs) per capita ou vendus ( 8 œufs) par ménage dans la présente étude le confirme.

Toutefois, ce constat est en contradiction avec les résultats rapportés par Mandal et al. (2006) en Inde. En effet, ces auteurs ont révélé que la majorité des propriétaires de volailles $(85 \%)$ utilisent les œufs pour la consommation domestique et que seulement $15 \%$ des répondants vendent les œufs excédentaires.
L'analyse des différentes activités pratiquées par les enquêtés montre que l'élevage avicole au sein du ménage est toujours associé à d'autres activités. En effet, l'agriculture au sens large pratiquée par la majorité des enquêtés corrobore les constats faits par Aboe et al. (2006) au Ghana et Moula et al. (2012) en RDC. D'après ces auteurs, l'association de l'aviculture familiale à d'autres activités agricoles surtout les cultures vivrières et de rente au sein du ménage permet aux chefs de ménage de faire face aux différents besoins quotidiens tant alimentaires que monétaires.

\section{Cheptel et facteurs de variation des effectifs de poules}

Le taux de mortalité des poules de $39,11 \%$ est jugé acceptable si nous nous référons aux valeurs d'au moins 60\% rapportées par Sarkar et Bell (2006). Nous nous accordons avec Nahimana et al. (2017) que cette situation pourrait s'expliquer par les différentes précautions prises par les aviculteurs pour protéger les poussins et les adultes en plus de la mise en œuvre du programme de vaccination et de l'encadrement des aviculteurs en matière de gestion de la santé et de l'alimentation de la volaille dont ils ont bénéficié. Ce résultat montre que des marges de progrès sont encore possibles pour soutenir l'aviculture familiale, notamment à travers des innovations technologiques.

Les causes de la mortalité des poules dans la présente étude sont identiques à celles rapportées par d'autres auteurs (Leta et Bakana, 2010 ; Nahimana et al., 2017). Les plus importantes ont été, en accord avec Nahimana et al. (2017), les prédateurs $(87,41 \%)$. La faible prévalence des maladies infectieuses et parasitaires $(8,21 \%)$ pourrait s'expliquer par le respect du programme de la vaccination et des conseils en rapport avec la santé de la volaille. Néanmoins, ce constat est en contradiction avec les résultats de Haoua et al. (2015) selon lesquels les maladies sont le principal facteur limitant l'essor de l'aviculture familiale en milieu rural des pays en voie de développement. 
Cette mortalité pourrait encore diminuer si les aviculteurs faisaient une bonne gestion de l'alimentation des oiseaux. En effet, certains auteurs (Tadelle et Ogle, 2001 ; Minh et al., 2004) ont rapporté que lorsqu'une poule est bien nourrie, elle est résistante aux maladies et aux parasites et les taux de croissance et de fécondité augmentent ainsi que les effectifs.

Le taux d'éclosion moyen $(81,46 \%)$ dans la présente étude qui se situe dans l'intervalle (4-100\%) et peut être jugé acceptable d'après Sonaiya et Swan (2004). Ces auteurs ont signalé qu'un taux d'éclosion supérieur à $75 \%$ est satisfaisant en couvaison naturelle. Nahimana et al. (2017) avaient fait les mêmes observations dans la même région. Toutefois, ce taux est inférieur à celui $(87 \%)$ observé par Sarkar et Bell (2006) au Bangladesh. Cette situation pourrait s'expliquer par les mesures prises par les aviculteurs pour sécuriser les poules en couvaison. En effet, pendant l'incubation, de l'eau ainsi qu'une ration équilibrée (30 à $50 \mathrm{~g}$ d'aliments) étaient placées à proximité du nid de la poule en couvaison chaque jour. Cette pratique diminue par conséquent le temps que la poule passe en dehors du nid à la recherche de la nourriture, ce qui améliore par la suite le taux d'éclosion.

Ce taux d'éclosion élevée pourrait également s'expliquer par le sex-ratio d'au plus 1 : 5 observé dans cette étude qui est de loin supérieur à celui (un coq pour dix poules) recommandé par van Eekeren et al. (2004) pour maximiser la fertilité.

Le faible effectif de coqs dans les ménages suivis pourrait être dû à l'encadrement des aviculteurs, ce qui faisait que les nouveaux coquelets adultes étaient directement consommés ou vendus. Par ailleurs, la présence de beaucoup de mâles adultes dans la basse-cour a un effet négatif sur la reproduction des femelles étant donné qu'ils passent plus de temps à se disputer les femelles qu'à assurer la monte (FAO, 2014).

L'observation des charges alimentaires annuelles par ménage (11287 FCFA), soit moins de 50 FCFA par jour, montre que les poules reçoivent très peu d'attention. Cette situation, en accord avec Kingori et al. (2010), oblige les oiseaux à se débrouiller pour se nourrir dans le milieu environnant caractérisé par l'irrégularité des ressources alimentaires picorables. Par ailleurs, Mwalusanya et al. (2002) ont rapporté que l'alimentation des poules locales est constituée par au moins $75 \%$ des issus de battages et différents produits picorés en divaguant. C'est pourquoi lorsque les compléments existent, ils ne sont distribués que sous forme de poignées sans tenir compte de l'effectif ou du stade physiologique (Halima et al., 2007 ; Muchadeyi et al., 2004). Par conséquent, ils couvrent difficilement les besoins d'entretien et ne répondent jamais aux besoins de production (FAO, 2014).

\section{Performances et profitabilité des poules en aviculture familiale dans un ménage}

Le rapport investissement sur profit de $1: 1,75$ si les consommations sont exclues et de $1: 4,05$ dans le cas contraire montre que l'aviculture familiale, bien que souvent requérant très peu d'intrants, est rentable (Goodger, 2002). D'après cet auteur, un rendement supérieur à 1 signifie que l'activité est rentable pour l'éleveur.

$\mathrm{Ce}$ rendement est supérieur aux résultats rapportés par Njue et al. (2004) au Kenya pour avoir fait la vaccination et la complémentation en même temps $(1,34)$, la complémentation seule $(1,15)$ ou la vaccination seule $(3,36)$. Il est aussi supérieur à celui (4) rapporté par Danho et al. (2004) lorsqu'ils avaient vacciné, déparasité et supplémenté tous les oiseaux. La supériorité de nos résultats pourrait s'expliquer par la mise en application de tous les conseils issus de l'encadrement bihebdomadaire des aviculteurs suivis en matière de gestion de la santé et de l'alimentation de la volaille. Elle pourrait également être due au nombre important d'élevages suivis et la durée de suivi dans la présente étude contrairement à au plus de 4 ménages et à la durée de suivi ( 8 mois) chez Danho et al. (2004).

Toutefois, il est inférieur à celui $(5,6)$ rapporté par ces derniers auteurs qui, en plus des interventions de déparasitage et de la 
vaccination des oiseaux de tout le cheptel, ont protégé tous les poussins contre les prédateurs jusqu'à trois mois d'âge et la complémentation faite tenait compte des besoins physiologiques des oiseaux, ce qui n'était pas le cas dans la présente étude. Cette différence pourrait également être due à la durée du suivi qui a été de huit mois chez ces derniers auteurs contrairement à notre cas (une année).

Donc, en tenant compte de ce rapport et considérant la petitesse des coûts de production, nos résultats confirment ceux de Guèye (2000) et Fasina et al. (2007) selon lesquels la commercialisation des produits avicoles constitue un bénéfice net pour les aviculteurs ruraux qui n'ont pas surtout accès à la terre et à d'autres ressources importantes comme les ruminants. Elle constitue aussi, en accord avec Alabi et al. (2006) et Aklilu et al. (2007), l'une des rares occasions pour les ruraux de générer rapidement des revenus en espèces afin de subvenir à leurs besoins.

Les revenus issus de la vente des poules sont utilisés dans différents achats et/ou dépenses familiales (vivres/aliments, intrants agricoles, condiments, habits/chaussures, fournitures scolaires, participation aux cérémonies familiales, soins de santé, cotisation en " tontine", économie familiale, ...). Donc, en accord avec Guèye (2003), les revenus générés par cette vente sont distribués directement ou indirectement pour le bien-être de tous les membres du ménage. Par ailleurs, certains auteurs (Mandal et al., 2006) avaient rapporté que la vente des poules a toujours un rôle important dans la satisfaction des besoins domestiques et économiques pour des millions de producteurs démunis.

La consommation moyenne annuelle d'œufs par individu de 8,1 œufs dans la zone d'étude est très faible si nous la comparons aux valeurs moyennes au niveau national de 40 œufs par personne et par an rapportées au Sénégal par FAO (2014) surtout constitués par les œufs de table provenant de l'élevage industriel. Le résultat de 8 œufs par individu en milieu rural est unique. Vu l'effectif du nombre d'œufs pondus et couvés et les œufs consommés annuellement par individu, les œufs produits en milieu rural sont quasiment destinés à la reproduction pour renouveler le cheptel avicole, ce qui confirme les constats faits par Sonaiya et Swan (2004), van Eekeren et al. (2004), FAO (2014) et Nahimana et al. (2017).

\section{Sécurité nutritionnelle des ménages ruraux et rôle des poules indigènes à Salémata (Sénégal)}

La consommation moyenne annuelle de 7,1 poules par personne et par an montre le rôle de l'aviculture familiale pour faire face à la malnutrition protéino-calorifique qui affecte la majorité des populations rurales. En effet, si nous considérons un rendement d'abattage des poules locales au Sénégal de $75 \%$ avec un poids moyen variant de $900 \mathrm{~g}$ à $2,4 \mathrm{~kg}$ (Ali, 2001), cette consommation per capita est d'au moins $7 \mathrm{~kg}$. Elle est supérieure à la moyenne du pays qui est de 5,5 $\mathrm{kg}$ (FAO, 2014) ou de $3,4 \mathrm{~kg}$ (Niang et Mbaye, 2013) et à celle de $3,4 \mathrm{~kg}$ observée en milieu rural sénégalais (FAO, 2014). Elle est presque égale à celle (7 $\mathrm{kg}$ ) observée par Sarkar et Bell (2006) au Bangladesh après des interventions visant à contrôler les maladies, à corriger la malnutrition des poules et à faire face aux faibles taux de croissance et de survie des poussins.

Les différentes occasions de la consommation des poules locales les plus importantes rencontrées dans la présente étude sont représentées par les festivités religieuses et de fin d'année comme Aklilu et al. (2004) l'avaient rapporté en Ethiopie et en Ouganda, respectivement. Cette situation serait favorisée aussi par le fait que la consommation de volaille notamment les poules ne connaît pas d'interdits religieux, contrairement à la viande porcine chez les musulmans et les chrétiens orthodoxes (Tadele et Ogle, 2001). C'est pourquoi cette large acceptabilité de la viande de volaille pourrait alors constituer une opportunité de développement de l'aviculture. Et, par conséquent, faire face à la demande accrue en volailles (Mack et al., 2005) causée par l'urbanisation croissante observée dans les pays en voie de développement. 
De ce qui précède, nous nous accordons avec Sarkar et Bell (2006) à dire que l'amélioration de la survie des poussins pourrait accroître l'effectif des poules consommées et/ou commercialisées.

Ainsi donc, en accord avec Scaners (2007) et Fasina et al. (2007), l'aviculture familiale contribue significativement à la lutte contre la pauvreté et au bien-être des aviculteurs ruraux vulnérables dont les femmes et les enfants.

\section{Conclusion}

Il ressort de cette étude que l'aviculture familiale contribue significativement à atténuer la pauvreté des ménages ruraux, à améliorer la sécurité alimentaire et l'approvisionnement des villes en poules. Il a été aussi constaté que, malgré le faible coût d'investissement, l'aviculture familiale parvient à générer plus de revenus. Ainsi, l'autosuffisance alimentaire ainsi que le rapport investissement/revenus pourraient être plus importants si des mesures d'amélioration de la protection des poussins, de l'alimentation et de la santé des oiseaux sont entreprises par les aviculteurs et les pouvoirs publics.

\section{CONFLITS D'INTERETS}

Les auteurs déclarent qu'il n'existe aucun conflit d'intérêts concernant la publication de cet article.

\section{CONTRIBUTIONS DES AUTEURS}

GN a contribué à la rédaction du protocole d'étude ; AM, SBA et WO ont contribué à son amélioration. Tous les auteurs ont participé à la rédaction du présent article.

\section{REMERCIEMENTS}

Les auteurs remercient le Projet de Développement de l'Elevage au Sénégal Oriental et la Haute Casamance (PDESOC) pour avoir mis à leur disposition les moyens nécessaires pour faciliter et mener à terme les enquêtes.

\section{REFERENCES}

Aboe PAT, Boa-Amponsem K, Okantah SA, Butler EA, Dorward PT, Bryant MJ. 2006. Free range village chickens on the Accra plains, Ghana: Their husbandry and productivity. Trop. Anim. Health Prod., 38: 235-248. DOI: 10.1007/s11250-006-4356-X

Aklilu HA, Almekinders CJM, Udo HMJ, Van Der Zijpp AJ. 2007. Village poultry consumption and marketing in relation to gender, religious festivals and market access. Trop. Anim. Health Prod., 39 (3): 165-177. DOI: 10.1007/s11250-0079002-8

Alabi RA, Esobhawan AO, Aruna MB. 2006. Econometric determination of contribution of family poultry to women's income in Niger-delta, Nigeria. J. Cent. Eur. Agric., 7 (4): 753-760. DOI: $10.5513 /$ jcea.v7i4.421

Ali D. 2001. Etude de l'influence du niveau énergétique de la ration sur la productivité de la poule locale (Gallus domesticus). (Thèse Méd. Vét.). Ecole Inter Etats des Sciences et Médecine Vétérinaires : Dakar, 2001, 20.

Benabdeljelil K, Arfaoui T. 2001. Characterization of Beldi chicken and turkeys in rural poultry flocks of Morocco. Current state and future outlook. AGRI, 31: 87-95. DOI: https://doi.org/10.1017/S10142339 00001516

Coplad JW, Alders RG. 2005: The Australian village poultry development programme in Asia and Africa. World's Poult. Sci. J., $\quad$ 61: 31-38. DOI: https://doi.org/10.1079/WPS20043 9

Danho T, Kacou A, Bodjo SC, CouacyHymannn E. 2004. Amélioration de la production en aviculture familiale: cas de la Côte d'Ivoire. In: Improving farmyard poultry production in Africa: Interventions and their economic assessment. Proceedings of a final research coordination meeting. FAO/IAEA Division of Nuclear Techniques in Food and Agriculture and 
held in Vienna, 24-28 May 2004, 107115

Dinka H, Chala R, Dawo F, Leta S, Bekana E. 2010. Socio-economic importance and management of village chicken production in rift valley of Oromia, Ethiopia. Livest. Res. Rural Dev., 22: 203.

http://www.lrrd.org/lrrd22/11/dink22203 .htm

Fall AK, Dieng A, Samba ANS, Diallo A. 2016. L'aviculture urbaine familiale au Sénégal: caractérisation et rôle socioéconomique dans la commune de Thiès. Rev. CAMES, 4 (2) : 6-11.

FAO. 2014. Secteur Avicole Senegal. Revues nationales de l'élevage de la division de la production et de la santé animales de la FAO. No. 7. Rome, 70p

Fasina FO, Mohammed SN, Onyekonwu ON, Wai MD. 2007. Contribution de l'aviculture aux revenus des ménages: le cas de la municipalité de Jos South (Nigeria). Aviculture Familiale, 17 (1 et 2) : 30-34.

Goodger WJ. 2002. Using Partial Budgets to assess if the interventions you designed/implemented make more than they cost. Proceedings of the 3rd scientific coordination meeting of FAO/IAEA of the coordinated research project on assessment of the effectiveness of vaccination strategies against Newcastle Disease and Gumboro Disease using immunoassay-based technologies for increasing farmyard poultry production in Africa. Quatre Bornes, Mauritius. May 6-10, 2002.

Guèye EF. 2003. Gender issues in family poultry production systems in lowincome food-deficit countries. American Journal of Alternative Agriculture, 18(4): $\quad 185-195 . \quad$ DOI: 10.1079/AJAA200350

Guèye EF. 2000. The role of family poultry in poverty alleviation, food security and the promotion of gender equality in rural Africa. Outlook on Agriculture, 29(2): 129-136.

DOI: $10.5367 / 000000000101293130$
Halima H, Neser FWC, Tadelle D, Van Marlekoster E, de Kock A. 2007. Village based indigenous chicken production system in north-west Ethiopia. Trop. Anim. Health Prod., 39: 189-197. DOI: 10.1007/s11250-007-9004-6

Haoua MT, Keambou CT, Poutougnigni MY, Manjeli Y. 2015. Characterisation of indigenous chicken production systems in the Sudano-sahelian zone of Cameroon. Livest. Res. Rural Dev., 27: 30.

http://www.lrrd.org/lrrd27/2/haou27030. html

Hofman A. 2000. Amélioration de l'aviculture traditionnelle aux îles Comores : Impact de la semi-claustration et de la complémentation par une provende locale sur la productivité de la volaille locale. Mémoire de $3^{\text {ème }}$ doctorat en Médecine Vétérinaire. FMV/Université de Liège : Liège, 71 pages

Kingori AM, Wachira AM, Tuitoek JK. 2010. Indigenous Chicken Production in Kenya: A Review. Int. J. Poult. Sci., 9 (4): 309-316. DOI: $10.3923 /$ ijps.2010.309.316

Leta S, Bekana E. 2010. Survey on Village Based Chicken Production and Utilization System in Mid Rift Valley of Oromia, Ethiopia. Global Veterinaria, 5 (4): 198-203. http://hdl.handle.net/Research/283

Mack S, Hoffmann D, Otte J. 2005. The contribution of poultry to rural development. World's Poult. Sci. J., 61 (1): $\quad$ 7-14. DOI: https://doi.org/10.1079/WPS20043 6

Mandal MK, Khandekar N, Khandekar P. 2006. Backyard poultry farming in Bareilly district of Uttar Pradesh, India: An analysis. Liv. Res. Rural Dev., 18: 101. http://www.lrrd.org/lrrd18/7/mand1 8101.htm

MEFP. 2014. Agence Nationale de la Statistique et de la Démographie (ANSD). Rapport Définitif du Recensement général de la population et de l'Habitat, de l'Agriculture et de 
l'Elevage (RGPHAE) : Etat et Structure de la population. Dakar/Sénégal, $70 \mathrm{p}$

Minh DV, Lindberg JE, Ogle B. 2004. Effect of scavenging and protein supplement on the feed intake and performance of improved pullets and laying hens in northern Vietnam. Asian-Aust. J. Anim. Sci., 17 (11): 1553-1561. DOI : 10.5713 / Ajas.2004.1553

Moula N, Detiffe N, Farnir F, AntoineMoussiaux N, Leroy P. 2012. Aviculture familiale au Bas-Congo, République Démocratique du Congo (RDC). Livest. Res. Rural Dev., 24: 74. http://www.lrrd.org/lrrd24/5/moul24074. $\mathrm{htm}$

Muchadeyi FC, Sibanda S, Kusina NT, Kusina J, Makuza S. 2004. The village chicken production system in Rushinga District of Zimbabwe. Livest. Res. Rural Dev., 16 :

40. http://www.lrrd.org/lrrd16/6/much16040. $\mathrm{htm}$

Mwalusanya NA, Katule AM, Mutayoba SK, Mtambo MMA. 2002. Productivity of local chickens under village management conditions. Trop. Anim. Health Prod., 34: 405-416. DOI: https://doi.org/10.1023/A:1020048327 158

Nahimana G, Missohou A, Ayssiwede SB, Cissé P, Butore J, Touré A. 2017. Amélioration de la survie des poussins et des performances zootechniques de la poule locale en condition villageoise au Sénégal. Rev. Elev. Med. Vet. Pays Trop., 70(1): 3-8. DOI: 10.19182/remvt.31393

Nahimana G, Missohou A, Ayssiwede SB. 2015. Influence de la durée d'élevage en claustration sur les performances zootechniques du couple mère-poussins de la poule locale au Senegal: cas du milieu contrôlé. Livest. Res. Rural Dev., 27:

152. http://www.lrrd.org/lrrd27/8/nahi27 152.html

Niang M, Mbaye M. 2013. Evolution des exportations de bétail malien au Sénégal suite aux récentes crises. Rapport final, Dakar/Sénégal, 37 p.

Njue SW, Kasiiti JL, Gacheru SG. 2004. Assessing the economic impact of commercial poultry feeds supplementation and vaccination against newcastle disease in local chickens, in Kenya. In: Improving farmyard poultry production in Africa: Interventions and their economic assessment. Proceedings of a final research coordination meeting. FAO/IAEA Division of Nuclear Techniques in Food and Agriculture and held in Vienna, 24-28 May 2004, 116136

Riise JC, Permin A, Kryger KN. 2005. Strategies for developing family poultry production at village level - Experiences from West Africa and Asia. World's Poult. Sci. J., 61: 15-22. DOI: 10.1079/WPS200437

Sarkar K, Bell JG. 2006. Potentiel du poulet indigène et son rôle dans la lutte contre la pauvreté et dans la sécurité alimentaire pour les ménages ruraux. Bulletin $R I D A F, 16$ (2): 16-28.

Scanes CG. 2007. Contribution of Poultry to Quality of Life and Economic development in the Developing World. Poult. Sci., 86(11): 2289-2290. DOI: 10.3382/ps.2007-86-11-2289

Sonaiya EB, Swan SEJ. 2004. Production en aviculture familiale: un manuel technique. Organisation des Nations Unies pour l'Alimentation et l'agriculture, FAO/Productions et Santé Animales, Rome, 134 p.

Tadelle D, Ogle B. 2001. Village poultry production systems in the central highlands of Ethiopia. Trop. Anim. Health Prod., 33 (6): 521-537. DOI: 10.1023/a: 1012740832558

Van Eekeren N, Maas A, Saatkamp HW, Verschuur M. 2004. L'aviculture à Petite Échelle dans les Zones Tropicales (4ème edition). Agrodok 4 : Wageningen, PaysBas ; 83 p. 